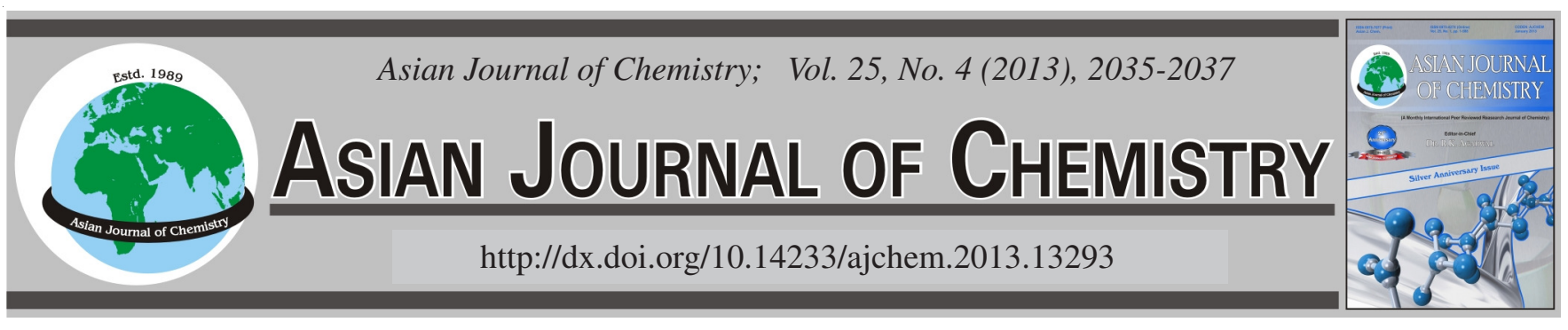

\title{
Synthesis and Antibacterial Activity of a Series of Heterocyclic Sulfonate Ligands and Their Metal Complexes
}

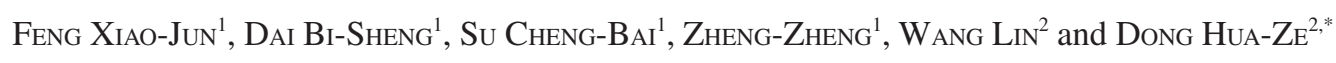

${ }^{1}$ Food and Drug Department, Qingyuan Polytechnic, Qingyuan 511510, P.R. China

${ }^{2}$ Department of Chemistry and Chemical Engineering, Hefei Normal University, Hefei 230061, P.R. China

*Corresponding author: E-mail: dapdong@163.com

(Received: 20 December 2011;

Accepted: 12 October 2012)

AJC-12273

A series of heterocyclic sulfonate ligands and their barium(II), silver(I), manganese(II), zinc(II) and cobalt(II) coordination compounds have been synthesized. Through antibacterial activities experiments of these complex compounds, metal salts as well as ligands, the rational conclusion is arrived that antibacterial activities of these compounds come from metal ions.

Key Words: Heterocyclic sulfonate ligand, Antibacterial activity, Coordination complex.

\section{INTRODUCTION}

Though organic sulfonate and their metal complexes have been studied by chemists ${ }^{1-8}$, in pharmacy, the antibacterial activities of these compounds are explored scarecely. To perfect coordination and broad-spectrum antibacterial ability, sulfonic acid is modified with pyrimidine.

\section{EXPERIMENTAL}

All the solvents and chemicals were used without further purification. Infrared spectra $\left(4000-400 \mathrm{~cm}^{-1}\right)$ were recorded with a Bruker Vector 22 FTIR spectrophotometer on KBr disks. Elemental analyses were done by using a Perkin-Elmer 1400C analyzer. The microbial strains were from Agricultural Culture Collection of China (ACCC).

Synthesis of $\mathbf{L}_{1-3}$ : The series of ligands \{4-pyridin-2-ylpyrimidine-2-sulfonate $\left(\mathbf{L}_{\mathbf{1}}\right)$, 4-pyridin-3-yl-pyrimidine-2sulfonate $\left(\mathbf{L}_{\mathbf{2}}\right)$, 4-pyridin-4-yl-pyrimidine-2-sulfonate $\left(\mathbf{L}_{\mathbf{3}}\right)$ \} were synthesized according to the literature method ${ }^{9-11}$ with synthetic route below (Scheme-I).

Synthesis of complexes: $\left[\mathrm{BaL}_{\mathbf{1}}\left(\mathrm{NO}_{3}\right)\left(\mathrm{H}_{2} \mathrm{O}\right)\right]_{\mathrm{n}}(\mathbf{1})$, $\left\{\left[\mathrm{Ba}\left(\mathbf{L}_{\mathbf{1}}\right)_{2}\left(\mathrm{H}_{2} \mathrm{O}\right)\right] \cdot 2 \mathrm{H}_{2} \mathrm{O}\right\}_{\mathrm{n}}(\mathbf{2}),\left[\mathrm{BaL}_{2}\left(\mathrm{H}_{2} \mathrm{O}\right)\left(\mathrm{ClO}_{4}\right)\right]_{\mathrm{n}}(\mathbf{3})$, $\left[\mathrm{Ag}_{3}\left(\mathbf{L}_{\mathbf{1}}\right)_{3}\left(\mathrm{H}_{2} \mathrm{O}\right)_{2}\right]_{\mathrm{n}}(\mathbf{4}),\left[\mathrm{Ag}_{3}\left(\mathbf{L}_{\mathbf{2}}\right)_{3}\left(\mathrm{H}_{2} \mathrm{O}\right)_{2}\right]_{\mathrm{n}}(\mathbf{5}),\left[\mathrm{Ag}_{3}\left(\mathbf{L}_{\mathbf{1}}\right)_{3}\left(\mathrm{H}_{2} \mathrm{O}\right)_{2}\right]_{\mathrm{n}}$ (6), $\left[\mathrm{Mn}\left(\mathbf{L}_{\mathbf{1}}\right)_{2} \cdot \mathrm{H}_{2} \mathrm{O}\right](\mathbf{7}),\left[\mathrm{Mn}\left(\mathbf{L}_{\mathbf{2}}\right)_{2} \cdot \mathrm{H}_{2} \mathrm{O}\right](\mathbf{8}),\left[\mathrm{Mn}\left(\mathbf{L}_{3}\right)_{2}\right.$. $\left.4 \mathrm{H}_{2} \mathrm{O}\right] \cdot 2 \mathrm{H}_{2} \mathrm{O}(\mathbf{9}),\left[\mathrm{Zn}\left(\mathbf{L}_{\mathbf{1}}\right)_{2} \cdot \mathrm{H}_{2} \mathrm{O}\right](\mathbf{1 0}),\left[\mathrm{Zn}\left(\mathbf{L}_{\mathbf{2}}\right)_{2} \cdot \mathrm{H}_{2} \mathrm{O}\right](\mathbf{1 1})$, $\left[\mathrm{Zn}\left(\mathbf{L}_{3}\right)_{2} \cdot 4 \mathrm{H}_{2} \mathrm{O}\right] \cdot 2 \mathrm{H}_{2} \mathrm{O}(\mathbf{1 2}),\left[\mathrm{Co}\left(\mathbf{L}_{\mathbf{1}}\right)_{2} \cdot \mathrm{H}_{2} \mathrm{O}\right](\mathbf{1 3}),\left[\mathrm{Co}\left(\mathbf{L}_{\mathbf{2}}\right)_{2} \cdot \mathrm{H}_{2} \mathrm{O}\right]$ (14), $\left[\mathrm{Co}\left(\mathbf{L}_{3}\right)_{2} \cdot 4 \mathrm{H}_{2} \mathrm{O}\right] \cdot 2 \mathrm{H}_{2} \mathrm{O}(\mathbf{1 5})$.

The single crystals of complexes 1-3 were used for detection of antibacterial activity directly (CCDC: 1, 760305;

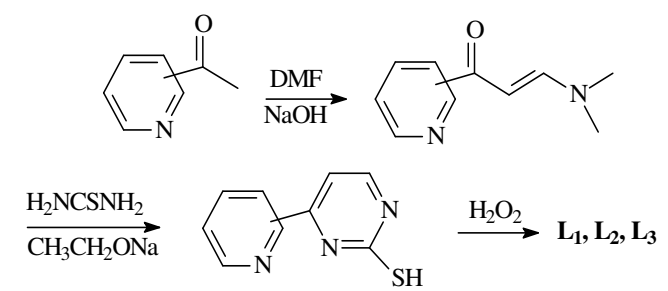<smiles>O=S(=O)(O)c1nccc(-c2ccccn2)n1</smiles>

$\mathbf{L}_{1}$<smiles>O=S(=O)(O)c1nccc(-c2cccnc2)n1</smiles>

$\mathbf{L}_{2}$<smiles>O=S(=O)(O)c1nccc(-c2ccncc2)n1</smiles>

$\mathbf{L}_{3}$
Scheme-I: Synthetic route of title ligands

2, 760306; 3, 760307). Synthesis, characterization, crystal data and structure were reported in earlier thesis ${ }^{12}$.

$\mathrm{NaL}_{1}, \mathrm{NaL}_{2}, \mathrm{NaL}_{3}(26.0 \mathrm{mg}, 0.1 \mathrm{mmol})$ were added, respectively in water $(5 \mathrm{~mL})$, then triplicate solutions of $\mathrm{AgNO}_{3}$ $(8.5 \mathrm{mg}, 0.05 \mathrm{mmol})$ in acetonitrile $(5 \mathrm{~mL})$ were very slowly dropped in. Crystal products $(\mathbf{4}, \mathbf{5}, \mathbf{6})$ formed after two-three weeks standing in darkness. The structures of $\mathbf{4 , 5}$ were confirmed by $\mathrm{Liu}$ and $\mathrm{Hu}^{13}$.

4: Yield $78 \%$. Anal. calcd. $(\%)$ for $\mathrm{Ag}_{3}\left(\mathbf{L}_{1}\right)_{3}\left(\mathrm{H}_{2} \mathrm{O}\right)_{2}$ $\left\{\left[\mathrm{Ag}_{3}\left(\mathrm{C}_{9} \mathrm{H}_{6} \mathrm{~N}_{3} \mathrm{O}_{3} \mathrm{~S}\right)_{3}\left(\mathrm{H}_{2} \mathrm{O}\right)_{2}\right]\right\}: \mathrm{C}, 30.35 ; \mathrm{H}, 2.08 ; \mathrm{N}, 11.80$. Found. (\%): C, 30.34; H,2.10; N, 11.77. Main IR absorption $\left(\mathrm{KBr}, v_{\max }, \mathrm{cm}^{-1}\right): 3431(\mathrm{~m}), 1615(\mathrm{~m}), 1578(\mathrm{~s}), 1533(\mathrm{~m})$, 1496 (m), 1385 (m), 1225 (s), 1197 (s), 1043 (s). 


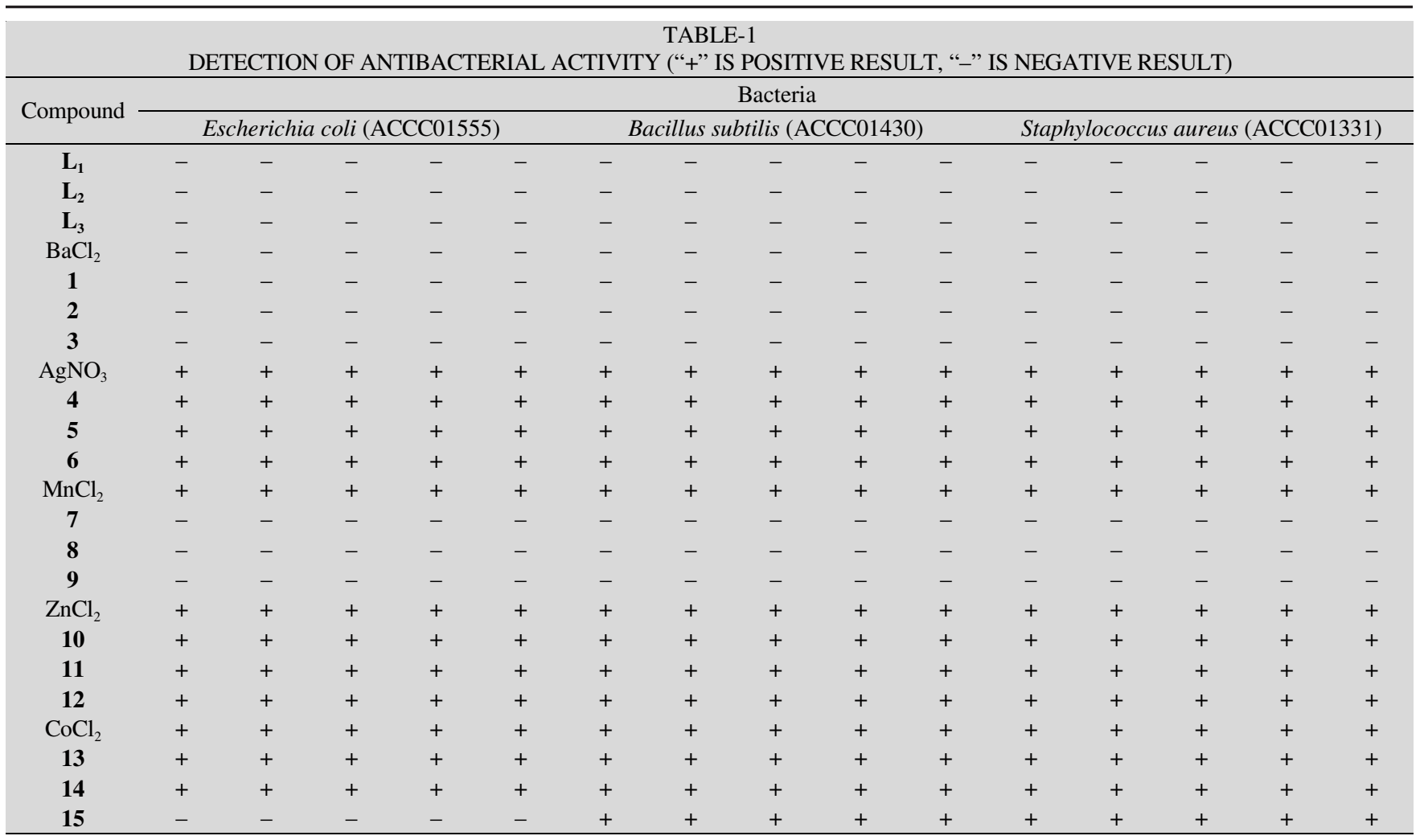

5: Yield $64 \%$. Anal. calcd. (\%) for $\mathrm{Ag}_{3}\left(\mathbf{L}_{2}\right)_{3}\left(\mathrm{H}_{2} \mathrm{O}\right)_{2}$ $\left\{\left[\mathrm{Ag}_{3}\left(\mathrm{C}_{9} \mathrm{H}_{6} \mathrm{~N}_{3} \mathrm{O}_{3} \mathrm{~S}\right)_{3}\left(\mathrm{H}_{2} \mathrm{O}\right)_{2}\right]\right\}: \mathrm{C}, 30.35 ; \mathrm{H}, 2.08 ; \mathrm{N}, 11.80$. Found (\%): C, 30.33; H, 2.10; N, 11.83. Main IR absorption $\left(\mathrm{KBr}, v_{\max }, \mathrm{cm}^{-1}\right): 3431(\mathrm{~m}), 1617(\mathrm{~m}), 1573(\mathrm{~s}), 1530(\mathrm{~m})$, 1495 (m), 1386 (m), 1225 (s), 1197 (s), 1044 (s).

6: Yield $71 \%$. Anal. calcd. (\%) for $\mathrm{Ag}_{3}\left(\mathrm{~L}_{3}\right)_{3}\left(\mathrm{H}_{2} \mathrm{O}\right)_{2}$ $\left\{\left[\mathrm{Ag}_{3}\left(\mathrm{C}_{9} \mathrm{H}_{6} \mathrm{~N}_{3} \mathrm{O}_{3} \mathrm{~S}\right)_{3}\left(\mathrm{H}_{2} \mathrm{O}\right)_{2}\right]\right\}: \mathrm{C}, 30.35 ; \mathrm{H}, 2.08 ; \mathrm{N}, 11.80$. Found. (\%): C, 30.37; H, 2.07; N, 11.78. Main IR absorption $\left(\mathrm{KBr}, v_{\max }, \mathrm{cm}^{-1}\right): 3432(\mathrm{~m}), 1615(\mathrm{~m}), 1574(\mathrm{~s}), 1533(\mathrm{~m})$, 1495 (m), 1381 (m), 1222 (s), 1195 (s), 1041 (s).

The preparation of complexes (7-15) with similiar way, the process as followed: the ligand $\left(\mathbf{L}_{1}-\mathbf{L}_{3}, 0.0472 \mathrm{~g}, 0.2 \mathrm{mmol}\right)$ in warm water $(15 \mathrm{~mL})$ and an aqueous solution $(10 \mathrm{~mL})$ of metal salt $(0.1 \mathrm{mmol})\left[\mathrm{MnCl}_{2} \cdot 4 \mathrm{H}_{2} \mathrm{O} ; \mathrm{Zn}\left(\mathrm{ClO}_{4}\right)_{2} \cdot 6 \mathrm{H}_{2} \mathrm{O}\right.$; $\left.\mathrm{Co}\left(\mathrm{ClO}_{4}\right)_{2} \cdot 6 \mathrm{H}_{2} \mathrm{O}\right]$ were mixed. The mixture was stirred for $1 \mathrm{~h}$, the crystals were obtained in filtrate when the solution cooled to room temperature. CCDC: 9, 681493; 12, 681494; 15, 681495. The general formulas of these complexes are $\left[\mathrm{M}(\mathrm{L})_{2}\right] \cdot \mathrm{H}_{2} \mathrm{O}(\mathrm{L}$ $\left.=\mathbf{L}_{1}, \mathbf{L}_{2}\right)^{14}$ and $\left[\mathrm{M}(\mathrm{L})_{2} \cdot 4 \mathrm{H}_{2} \mathrm{O}\right] \cdot 2 \mathrm{H}_{2} \mathrm{O}\left(\mathrm{L}=\mathbf{L}_{3}\right)^{15}$. The structures of complexes 7, 10, 13 were reported by Dong et al. ${ }^{14}$.

8: Yield $86 \%$. Anal. calcd. (\%) for $\mathrm{C}_{18} \mathrm{H}_{14} \mathrm{MnN}_{6} \mathrm{O}_{7} \mathrm{~S}_{2}$ $\left\{\left[\mathrm{Mn}\left(\mathbf{L}_{2}\right)_{2}\right] \cdot \mathrm{H}_{2} \mathrm{O}\right\}: \mathrm{C}, 40.99 ; \mathrm{H}, 2.29 ; \mathrm{N}, 15.93$. Found. (\%): C, 40.97; H, 2.28; N, 15.89. Main IR absorption $\left(\mathrm{KBr}, \mathrm{v}_{\max }, \mathrm{cm}^{-1}\right)$ : 3442 (vs), 1616 (w), 1577 (s), 1534 (m), 1430 (m), 1418 (m), 1370 (w), 1249 (vs), 1197 (s), 1034 (s).

11: Yield $63 \%$. Anal. calcd. (\%) for $\mathrm{C}_{18} \mathrm{H}_{14} \mathrm{ZnN}_{6} \mathrm{O}_{7} \mathrm{~S}_{2}$ $\left\{\left[\mathrm{Zn}\left(\mathbf{L}_{2}\right)_{2}\right] \cdot \mathrm{H}_{2} \mathrm{O}\right\}: \mathrm{C}, 38.89 ; \mathrm{H}, 2.54 ; \mathrm{N}, 15.12$. Found. (\%): C, $38.85 ; \mathrm{H}, 2.53 ; \mathrm{N}, 15.10$. Main IR absorption $\left(\mathrm{KBr}, \mathrm{v}_{\max }, \mathrm{cm}^{-1}\right)$ : 3445 (vs), 1614 (w), 1579(s), 1533(m), 1432(m), 1416(m), 1374(w), 1246(vs), 1199(s), 1034(s).

14: Yield $62 \%$. Anal. calcd. (\%) for $\mathrm{C}_{18} \mathrm{H}_{14} \mathrm{CoN}_{6} \mathrm{O}_{7} \mathrm{~S}_{2}$ $\left\{\left[\mathrm{Co}\left(\mathbf{L}_{2}\right)_{2}\right] \cdot \mathrm{H}_{2} \mathrm{O}\right\}: \mathrm{C}, 39.35 ; \mathrm{H}, 2.57 ; \mathrm{N}, 15.30$. Found. (\% ): C, 39.33 ; H, 7.59; N, 15.32. Main IR absorption $\left(\mathrm{KBr}, \mathrm{v}_{\max }, \mathrm{cm}^{-1}\right)$ :
3448 (vs), 1617 (w), 1574 (s), 1531 (m), 1434 (m), 1420 (m), 1372 (w), 1245 (vs), 1196 (s), 1032 (s).

Detection of antibacterial activity: After materials for detection of antibacterial activity antoclaved, each petri dish was infused proper nutrient agar $c a .40^{\circ} \mathrm{C}$. When the nutrient agar was cooled to room temperature, a solution of bacterial and a piece of circular paper $(0.5 \mathrm{~cm}$ in diameter) carrying with sample were placed on it in order. Then petri dish were put into electro-thermal incubator at $36^{\circ} \mathrm{C}$ for $24 \mathrm{~h}$. The control group $(n=5)$ was established with every compound. At the same time, a blank control group $(\mathrm{n}=5)$ without any sample was cultured.

As good water-solubility of $\mathbf{L}_{1-3}$ and inorganic compounds, their solutions $(0.001 \mathrm{~g} / \mathrm{mL})$ were used in detecting antibacterial activity. The rest of samples (1-15) were performed in solid on watery papers for their poor solubility. The positive result is that the inhibition zone was present and vice versa. The blank control group assumed negative result.

\section{RESULTS AND DISCUSSION}

Three kinds of bacterias are not inhibited by ligends $\left(\mathbf{L}_{1-3}\right)$ and anions $\left(\mathrm{Cl}^{-}, \mathrm{ClO}_{4}^{-}, \mathrm{NO}_{3}^{-}\right)$. Complexes and corresponding metal ions exhibits similar antibacterial activity. Enough metal ionic concentration is the cause of antimicrobial activity expect

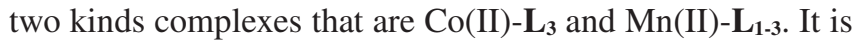
possible that dissociation constant of this two kinds metal complexes is too low to inhibit increase of microbe. These coordination compounds are potential sustained-release antibacterial drugs for their low dissociation constant.

\section{ACKNOWLEDGEMENTS}

This work was financially supported by Bureau of Science and Technology, Qingyuan (Project NO.2010A006, Project 
No. 2009B057). It is also supported by the National Science Foundation of China (No. 21101053), Program for Excellent Young Talents in University of Anhui Province (2011SQRL128), Anhui Provincial Natural Science Research Project (kj20112311) and Key Disciplines Foundation of Hefei Normal University.

\section{REFERENCES}

1. B.J. Guanderman and P.J. Squattrito, Inorg. Chem., 33, 2924 (1994).

2. E.J. Kosnic, E.L. McClymont, R.A. Hodder and P.J. Squattrito, Inorg. Chim. Acta, 210, 143 (1992).

3. A.J. Shubnell, E.J. Kosnic and P.J. Squattrito, Inorg. Chim. Acta, 216, 101 (1994)

4. S. Marder, J. Peny and C. Yakymyshyn, Chem. Mater., 6, 1137 (1994).

5. V. Russell, M. Eter and M. Ward, Chem. Mater., 6, 1206 (1994).

6. J. Cai, C.H. Chen, C.Z. Liao, X.L. Feng and X.M. Chen, Acta Crystallogr., B57, 520 (2001).
7. A.P. Cöté and G.K.H. Shimizu, Coord. Chem. Rev., 245, 49 (2003).

8. J.W. Cai, Coord. Chem. Rev., 248, 1061 (2004)

9. M.M. Bio, F. Xu, M. Waters, J.M. Williams, K.A. Savary, C.J. Cowden, C. Yang, E. Buck, Z.J. Song, D.M. Tschaen, R.P. Volante, R.A. Reamer and E.J.J. Grabowski, J. Org. Chem., 69, 6257 (2004).

10. H.B. Zhu, H.Z. Dong, W. Huang and S.H. Gou, J. Mol. Struct., 831, 55 (2007).

11. X.B. Fang, H.Z. Dong and D.B. Tian, Chin. J. Inorg. Chem., 25, 47 (2009).

12. H.-Z. Dong, J.-H. Bi, H.-B. Zhu and S.-H. Gou, J. Mol. Struct., 975 , 173 (2010).

13. X. Liu and D. Hu, Acta Cryst., E67, m1067 (2011).

14. H.B. Zhu, H.Z. Dong, W. Huang and S.H. Gou, J. Mol. Struct., 831, 55 (2007).

15. X.-B. Fang, H.-Z. Dong and D.-B. Tian, Chin. J. Inorg. Chem., 25, 47 (2009). 